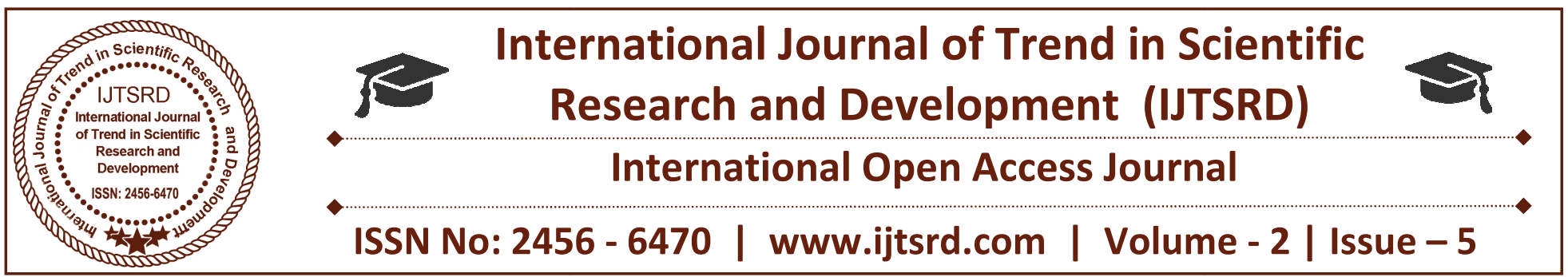

\title{
MAC: Protocols for Cognitive Radio Sensor Networks and their Comparision
}

\author{
Anupam Mittal, Geetika Sharma \\ CSE, Chandigarh University, \\ Gharuan, Punjab, India
}

\section{ABSTRACT}

Wireless Sensor Networks (WSNs) deliver smart communication paradigm which can set up intelligent networks handling all the applications as per the user requirements. The WSNs offer several advantages like flexibility, fault tolerance, low cost and easy deployment. Typically, a WSN consists of spatially distributed autonomous sensors to monitor physical or environmental conditions. These sensors generate report over the network on the occurrence of any event. For a WSN to provide better reliability and improved lifetime, an efficient Medium Access Control (MAC) protocol design holds a paramount importance. Several multichannel MAC protocols exist which performs better than the single channel protocols. The MAC layer coordinates transmission between the users sharing a spectrum and improves the throughput and energy efficiency. Cognitive Radio (CR) is an adaptive, intelligent radio and network technology that can automatically detect available channels in a wireless spectrum and make those channels available for concurrent transmissions. Dynamic spectrum access in the form of cognitive radio provides spectrum efficient communication for all WSNs which led to the development of Cognitive Radio Sensor Networks (CRSNs). This paper highlights an intensive survey of MAC protocols defined for WSNs and the design of Cognitive Radio based WSNs.

KEYWORD: cognitive radio wireless sensor network; wireless communication; wireless sensor network; energy efficiency; medium access control protocol; cognitive MAC

\section{INTRODUCTION}

In recent year, the most emerging and growing field of research and development is "Internet of Things" (IoT). It's a third revolution in which we are trying to connect the physical world with the imaginary world of electronics. Additionally, ubiquitous objects with cognitive capabilities will be able to make intelligent decisions [1]. The main motivating application behind this is to automatically connect, monitor and respond to nature's surveillance systems. This is due to the development of Wireless Sensor Network (WSN), in which traffic is always in burst mode. However, too many wireless technologies and equipment, such as interphone, WLAN, WPAN, RFID, Wireless USB, Bluetooth, WI-FI, Zig Bee/802.15.4 and so on, use the same unlicensed ISM band and make it overcrowded which affect directly on to the spectrum utilization as well as on energy efficiency. So, it's required to solve the problem of spectrum utilization in presence of other equipment. Cognitive radio (CR) improve version of software-defined radio, has been proposed to overcome the bandwidth limitations by the effective utilization of the spectrum by exploiting the existence of white space/spectrum hole [2], which is defined as the channels that are unused at a specific locations and time by its primary users (PUs). Integrating CR technology with wireless sensor (WS) node (i.e., Cognitive Radio-based Wireless Sensor Networks (CR-WSNs)), can help to overcome bandwidth limitation of WSNs by sensing spectrum hole and utilize that to improve the spectrum utilization and minimize interference with coexisting wireless technologies. But due to the characteristics and limitation of sensor nodes, the coupling of cognitive technology in sensor nodes introduce some more challenges and need to handle some additional 
task such as spectrum sensing, spectrum sharing, and spectrum management [3].

\section{COGNITIVE \\ RADIO \\ WSN \\ ARCHITECTURE}

CR-WSN is a network of WS nodes with an extra feature of cognitive capabilities. According to Joshi G. P. et al., CR-WSNs consist of many spatially distributed energy-constrained, self-configuring, selfaware WS nodes with cognitive technology [3]. They are not only using the white space/idle channel but also protect the rights of primary users (PUs), provide opportunistic channel access to WS nodes, dynamic spectrum access, improve the energy efficiency and reduce the overall delay. White spaces can be used as interweave, underlay or overlay patterns. In interweave pattern, CR nodes occupy the free space when PUs are idle. In the underlying paradigm, CR nodes use the licensed band using low-power to the limited range and it's in the present or absent of PUs. Both the SUs and PUs can transmit at the same time till it does not affect the transmission of PUs or up to some limit. Whereas in the overlay model, CR users use the licensed bands along with the PUs in cooperation manner by changing its communication characteristics, Figure 1 shows the CR-WSN architecture. CR-WSNs can be modelled as Ad Hoc CR-WSN, Clustered CR-WSN, Heterogeneous and Hierarchical CR-WSN or Mobile CR-WSN. For the comparison of ordinary WSNs, Ad Hoc CRNs and CR-WSNs refer [6]. In CR-WSN, communication unit having a modification of CR capabilities using which the sensor node alters its transmission parameters such as carrier frequency, transmission power, and modulation. The CR-WS nodes have mainly four functionalities: spectrum sensing, spectrum management, spectrum sharing, spectrum mobility [10]. Instead of paying for the expensive licenses, CRWSNs can offer wireless services by investing the comparatively small amount of capital in their infrastructure, and spectrum sensing technologies [6]. Country wise spectrum incompatibility problem can be solved. It can provide financial advantages to the PUs by renting or leasing their license spectrum band if underutilized. The probability of detection is the main metric to evaluate the Quality of Services (QoS). CR-WSNs have same hardware limitation as conventional WSNs. There may be frequently changed in the topology of the network due to the PUs activity. One most challenging issue is the channel selection in CR-WSNs. Due to limited energy; the energy consumption is also an essential design issue in CR-WSNs.

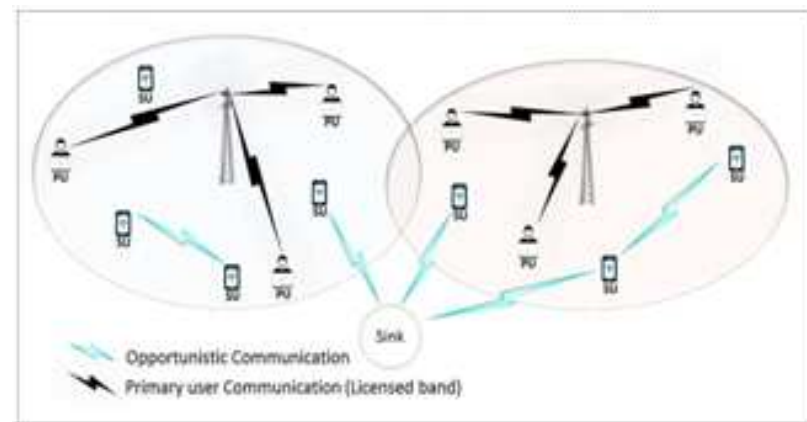

Fig.1. A typical cognitive radio wireless sensor network (CR-WSN) architecture.

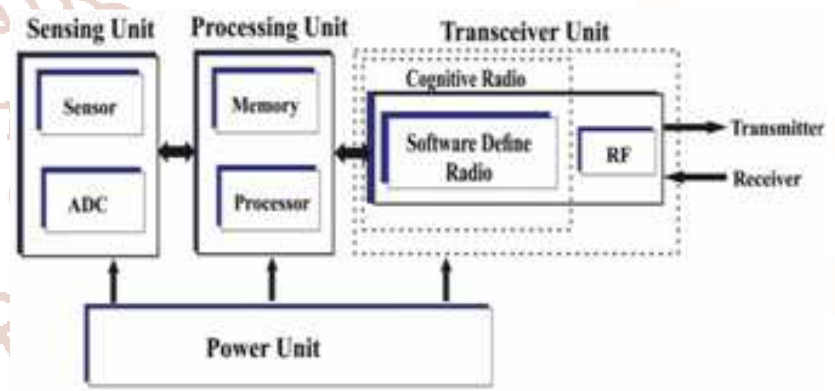

Fig -2: hardware structure of CR-WSN

\section{MEDIUM ACCESS PROTOCOLS IN CR-WSNS}

CONTROL

The MAC protocol improves energy efficiency in wireless communicating sensor networks by increasing sleep duration and decreasing all the sources of energy wastage. The major sources of energy waste are collision, overhearing, control packet overhead and idle listening. A MAC protocol reduces the waste of energy from all these sources. The functions of a good MAC protocol include framing, medium access, reliability, flow control and error control. Several MAC schemes have been proposed over the years to improve the overall performance of WSNs. These MAC protocols can be broadly classified as schedule based and Contention based schemes. The schedule based protocols involve time synchronization requirements but the contention based protocols relax time synchronization requirements. All these MAC protocols consist of acknowledgement (ACK) messages and retransmissions through which better reliability can be achieved.

\section{A. Sensor Mac (S-Mac) Protocol}

The Sensor-Media Access Control (S-MAC) is a contention based MAC protocol explicitly designed for wireless sensor networks. While reducing energy 
consumption is the primary goal in this design, the protocol also has good scalability and collision avoidance capability [1]. A combined scheduling and contention scheme is utilized in this protocol which helps in achieving good scalability and collision avoidance. The SMAC applies message passing to reduce the contention latency for sensor-network applications that require store-and-forward processing as data move through the network [1]. Energy consumption is reduced and self configuration is supported by three novel techniques in S-MAC. The energy waste is reduced to a great extent in S-MAC design. Here the time frame is divided as a listening session and a sleeping session. In SMAC, the sensor nodes communicate with additional nodes by sending some control packets such as SYNC, RTS (Request to send), CTS (Clear to Send) and ACK (Acknowledgement) during listen period. A SYNC packet exchange can synchronize all the nearest nodes collectively. Then the RTS/CTS switch is used over the two nodes that need to communicate with each other.
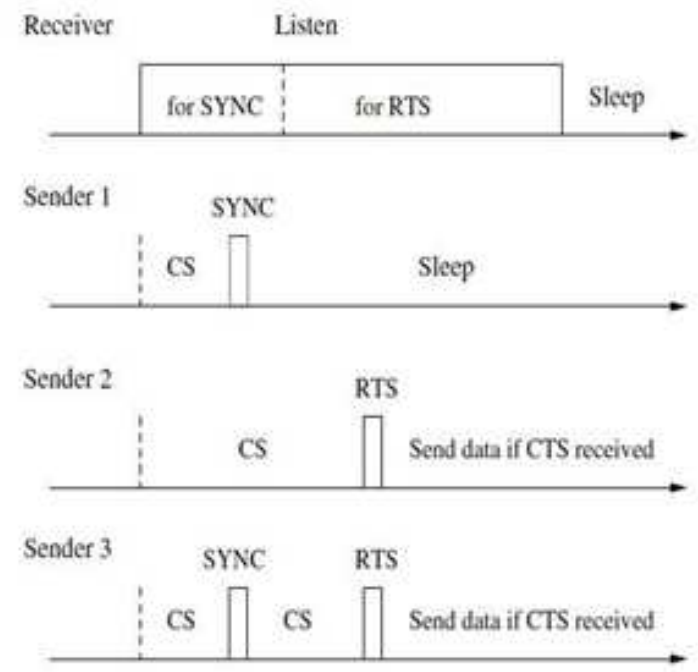

Fig 3: Timing relationship between a receiver and different senders, CS stands for carrier sense [1]

\section{B. Berkeley Mac (B-Mac) Protocol}

Another contention based MAC protocol extensively used in WSNs is the Berkeley Media Access Control (B-MAC) protocol. This scheme provides a flexible interface to obtain ultra low power operation, effective collision avoidance and high channel utilization [2]. An adaptive preamble sampling scheme is employed in B-MAC to achieve low power operation, reduced duty cycle and minimized idle listening. B-MAC supports on-the-fly reconfiguration and provides bidirectional interfaces for system services to optimize performance, whether it be for throughput, latency, or power conservation [2]. He design and implementation of B-MAC is very simple. A filter mechanism is used in B-MAC to increase reliability and channel assessment. B-MAC uses clear channel assessment (CCA) and packet back offs for channel arbitration, link layer acknowledgments for reliability and low power listening (LPL) for low power communication [2].

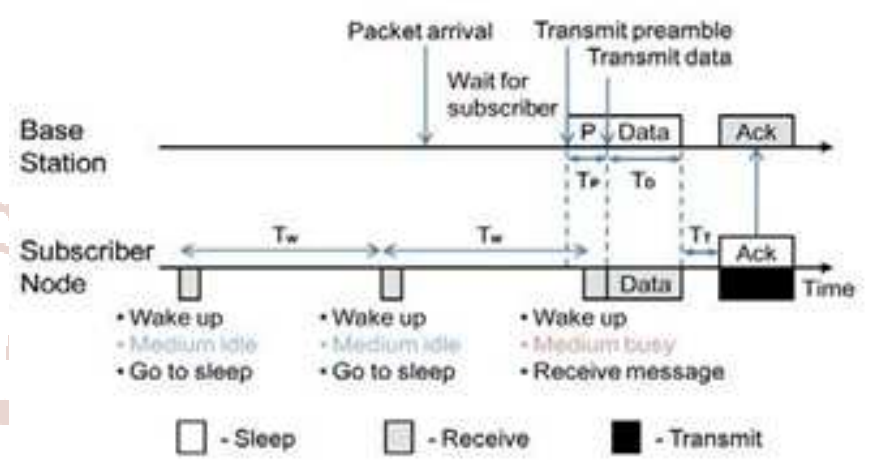

Fig 4: Preamble sampling in B-MAC

B-MAC uses CCA to decide the arrival of a packet on the wake up of nodes. No synchronization, RTS and CTS are there in B-MAC. When B-MAC is compared with $/$ another conventional S-MAC,B-MAC's flexibility results in better packet delivery rates, throughput, latency, and energy consumption than SMAC [2].

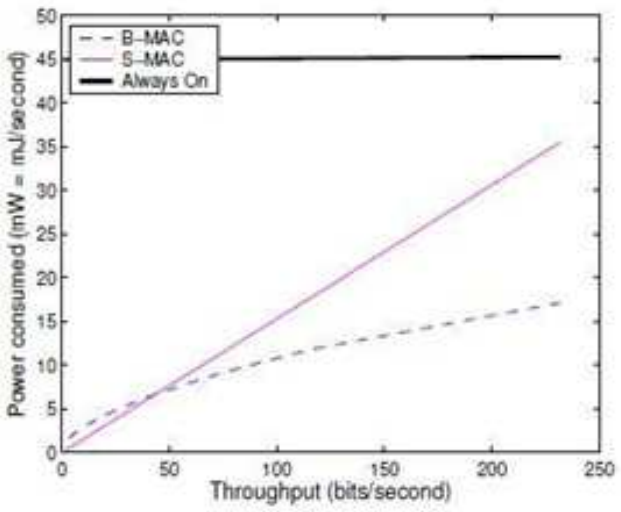

Fig 5: Comparison of B-MAC and S-MAC for power consumption in a 10-node Network [2]

\section{Y-Mac : Multi-Channel Mac Protocol}

The single channel MAC protocols have very limited performance in a multi-channel environment. Therefore several multi-channel MAC protocols have been proposed to improve the overall network performance. Y-Media Access Control SS(Y-MAC) protocol is one of the energy efficient MAC protocol for WSNs. The goal of this protocol is to achieve both 
high performance and energy efficiency under diverse traffic conditions [3]. Y-MAC protocol is a TDMAbased multi-channel MAC protocol [3]. So a time slot is allocated in Y-MAC for both data transmission and data reception. The Y-MAC achieves good reception rate, low duty cycles and a steady delivery latency even under high traffic conditions, while the other single channel MAC protocols suffer due to limited reception bandwidth. Y-MAC achieves effective transmission of bur sty messages under high traffic conditions, while maintaining low energy consumption [3]. The use of multiple channels can definitely increase MAC protocol performance with low energy consumption [3].

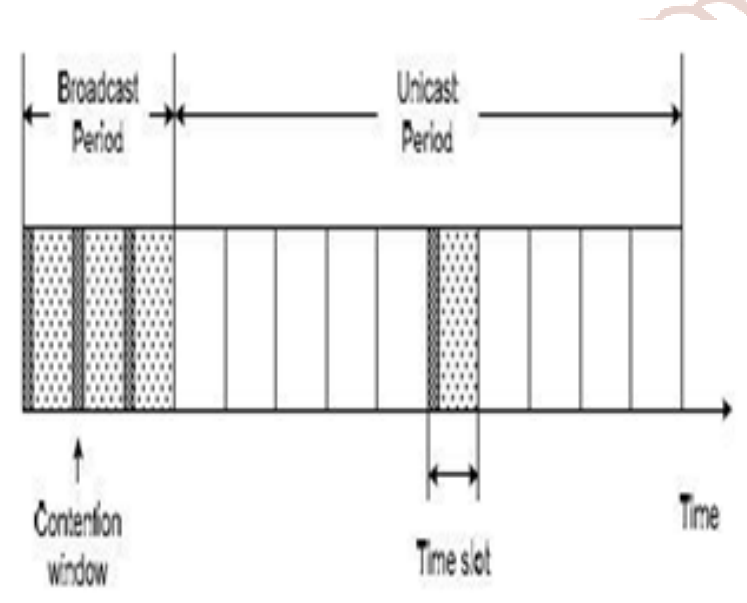

Fig 6: Frame architecture of Y-MAC [3]
In Y-MAC frame architecture, the time is divided into several fixed -length frames, and each frame is composed of a broadcast period and a uncast period [3]. The sender and receiver should have synchronization on the communication channel and transmission timing. Y-MAC can be implemented in the RETOS operating system running on Tmote Sky motes for performance analyzes. RETOS is an operating system for WSNs developed at Yonsei University which has a layered network architecture. It consists of three layers: the dynamic networking layer (DNL), the networking supporting layer (NSL), and the medium access control (MAC) layer [3]. User applications need only to interact with the DNL to deliver messages, and different routing modules can be selected taking into account the characteristics of user applications [3]. The NSL takes care of neighbourhood management and the neighbourhood information is also maintained in a table. RETOS is thus a multi-threaded operating system, so when a node receives a message it wakes up a blocked thread [3].
Extensive experiments validate the practicality of YMAC protocol in improved throughput and reduced message delivery latency. On comparing the multichannel energy efficient Y-MAC protocol with an another energy efficient MAC protocol defined for dense WSNs called Crankshaft, Y-MAC outperforms Crankshaft in terms of data reception rate.

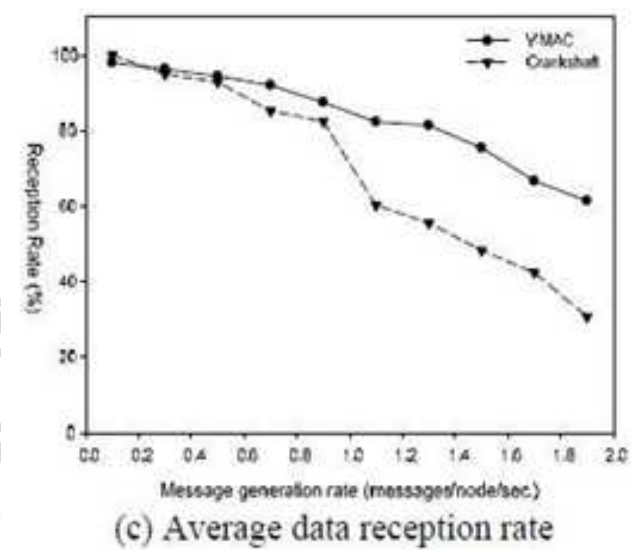

Fig 7: Comparison of Y-MAC and Crankshaft for data reception rate in multi-channel Environment [3]

\section{Cognitive Mac (C-Mac) : A Multi-Channel Mac Protocol}

The emergence of Cognitive Radio (CR) has become an ultimate solution for spectrum scarcity in wireless applications. Here another multi-channel MAC protocol, Cognitive Media Access Control (C-MAC) is introduced for $\mathrm{CR}$ sensor networks. C-MAC operates over multiple channels, and hence is able to effectively deal with, among other things, the dynamics of resource availability due to primary users and mitigate the effects of distributed quiet periods utilized for primary user signal detection [4]. In CMAC, each channel is logically divided into recurring super frames which, in turn, include a slotted beaconing period (BP) where nodes exchange information and negotiate channel usage [4]. A beacon is transmitted by each node in a destined beacon slot during the BP. The hidden nodes, medium reservations and mobility can be handled with this beacon.

In this scheme, a Rendezvous Channel (RC) is used as the backbone of C-MAC. The C-MAC protocol employs the concept of dynamic Rendezvous Channel (RC), which is used to coordinate nodes in different channels, for multi-channel resource reservation, quiet period (QP) coordination for incumbent detection, and so on. For making $\mathrm{RC}$ extremely robust to Incumbents, Backup Channel (BC) concept is also 
introduced here. C-MAC addresses many key challenges for the proper operation of WSNs in presence of incumbents. RC can achieve some features to manage the entire network like network wide Group Communication (GC), inter-channel synchronization, neighbourhood discovery and load balancing. C-MAC has been evaluated via both analytically and through simulations.

C-MAC has a unique channel structure where each channel has its own super frame structure. One of the available channels is identified as the RC. The other multi-channel MAC protocols use a super frame only in the common channel which requires the switching back of all network devices to the common channel on the start of every super frame. The maximum achievable throughput in C-MAC has been evaluated for number of channels and the result shows the effectiveness of C-MAC over multiple channels [4].

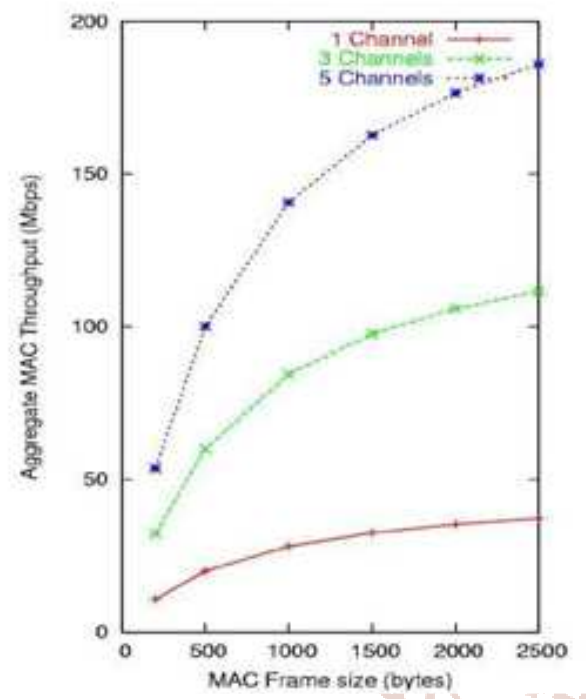

Fig 8: Maximum C-MAC aggregate throughput Above the MAC layer [4]

\section{E. Mmdqs-Mac: Multi hop Multi-Channel Distributed Qos Scheduling Mac}

The Multi hop Multi-Channel Distributed QoS Scheduling Media Access Control (MMDQS-MAC) scheme improves the network performance of WSNs by selecting the best channel for an individual wireless sensor node [5]. The overall network performance of WSNs is increased here by decreasing the chance of collisions and interferences. MMDQS MAC protocol utilizes parallel transmissions and also supports short packet transmissions under low traffic networks. This scheme improves the performance of aggregate throughput, probability of successful transmission, packet delivery ratio, energy consumption and average end-to-end delay [5].

When the medium is busy, all available multichannels are utilized to reduce the channel access time of a sensor node and hence improve the WSNs performances. The multi-channel MAC schemes employed here increase the capacity of wireless access control mechanisms. These multi-channel access mechanisms maintain different transmissions in wireless links active at the same time without collision. Such multi-channel scheduling MAC assignment can eliminate the interference among different channels and therefore, no collision in the MAC layer. In this protocol, each sensor node is equipped with directional antennas and the size of MAC layer packet is very small [5].

The primary objectives of MMDQS-MAC are maximum aggregate throughput, interference-free communication and guaranteed QOS. There are three assumptions regarding the sensor nodes used in this protocol: All sensor nodes are equipped with directional antennas, all sensor nodes are stationary and Sensor nodes can choose arbitrary transmits power for data transmission [5].

The frame structure of MMDQS -MAC protocol consists a Contention Period (CP) and a Non Contention Period (NCP). The beacon frames are broadcasted during $\mathrm{CP}$ to perform time synchronization periodically.

According to G. A. Shah and et al., Cognitive Adaptive MAC (CAMAC) protocol which works on mainly three tasks to improve spectrum utilization and energy efficiency: (i) On-demand spectrum sensing; (ii) Limiting the number of spectrum sensing nodes; and (iii) Applying a duty cycle [20]. In this proposed protocol, fast sensing is to reduce time delay and fine sensing to protect the rights of PU. CAMAC operated in three phases: Spectrum Measurement phase, CCP and DTP. In this paper [21], authors have described the case where the WLAN and WSN are operated in the same region. WSN packets always lost in existence of WLAN. But WLAN traffic is not always continuing in nature rather its bur sty with long white space. So, it is feasible to utilize the same hole by the CRSN to operate in co-existence. COG-MAC is the extended version of carrier sense based MAC. In this article [22], authors have suggested a protocol that dynamically adjusts the channel negotiation period according to the network density. In many CCC based 
protocol suffers from $\mathrm{CCC}$ bottleneck problem negotiation $(\mathrm{CN})$ window is a bottleneck in a dense (Saturation of CCC) due to channel utilization network environment and large CN window size limitation, bandwidth waste in channel negotiation increase the time delay in a sparse network. and long channel access delay. Small channel

Table1. Comparison of MAC protocols

\begin{tabular}{|c|c|c|c|c|c|c|}
\hline $\begin{array}{l}\text { Ref. } \\
\text { Paper }\end{array}$ & $\mathrm{CCC}$ & $\begin{array}{l}\text { No. of } \\
\text { Tx-Rx }\end{array}$ & $\begin{array}{l}\text { Performance } \\
\text { Improvement }\end{array}$ & $\begin{array}{l}\text { Channel } \\
\text { Access }\end{array}$ & Description \& Advantages & Limitation \\
\hline [15] & No & Single & $\begin{array}{l}\text { Low Computational } \\
\text { Complexity\& } \\
\text { Delay }\end{array}$ & POMDP & $\begin{array}{l}\text { Long and short sensing Backup } \\
\text { channel based }\end{array}$ & $\begin{array}{l}\text { Extra energy } \\
\text { consumption } \\
\text { in backup } \\
\text { channel }\end{array}$ \\
\hline [16] & Yes & Single & $\begin{array}{l}\text { Bandwidth } \\
\text { Energy efficien }\end{array}$ & CSMA & $\begin{array}{l}\text { CSMA-based MAC protocol for } \\
\text { CCC Bandwidth efficient Low } \\
\text { packet delay }\end{array}$ & $\begin{array}{l}\text { Cannot } \\
\text { achieve the } \\
\text { QoS } \\
\text { requirement } \\
\text { of sensor } \\
\end{array}$ \\
\hline$[17]$ & No & Single & En & $\begin{array}{l}\text { Channel } \\
\text { Weight }\end{array}$ & $\begin{array}{l}\text { Protection from multichannel } \\
\text { hidden terminal problem } \\
\text { Cooperative sensing High } \\
\text { Throughput Lower packet loss }\end{array}$ & $\begin{array}{l}\text { It just sense } \\
\text { fewer } \\
\text { channels }\end{array}$ \\
\hline$[18]$ & Yes & Sing & Energy effici & $\begin{array}{l}\text { Energy } \\
\text { level }\end{array}$ & $\begin{array}{l}\text { High throughput and QoS. } \\
\text { Periodic channel sensing Duty } \\
\text { cycle based } \\
\text { and }\end{array}$ & $\begin{array}{l}\text { No } \\
\text { advantage in } \\
\text { increasing } \\
\text { the number } \\
\text { of } \\
\text { retransmissio } \\
\text { ns }\end{array}$ \\
\hline [19] & No & Tw & Low overl & $\begin{array}{l}\text { Energy } \\
\text { level }\end{array}$ & $\begin{array}{l}\text { A receiver based approach } \\
\text { Efficient and reliable Improve } \\
\text { frequency agility Broadcast-based }\end{array}$ & $\begin{array}{l}\text { Extra energy } \\
\text { consumption } \\
\text { in broadcast }\end{array}$ \\
\hline$[20]$ & Yes & Single & $\begin{array}{l}\text { Energy } \\
\text { Delay }\end{array}$ & $\begin{array}{l}\text { Slotted } \\
\text { ALOHA }\end{array}$ & $\begin{array}{l}\text { On-demand spectrum sensing } \\
\text { Limited number of sensing nodes } \\
\text { Fast and fine sensing }\end{array}$ & $\begin{array}{l}\text { Energy } \\
\text { consumption } \\
\text { is high for } \\
\text { sensing } \\
\text { nodes }\end{array}$ \\
\hline$[21]$ & No & Single & Energy efficient & CSMA & $\begin{array}{l}\text { Single hop distance optimization } \\
\text { approach Energy efficient Packet } \\
\text { size optimization }\end{array}$ & $\begin{array}{l}\text { High } \\
\text { Complexity }\end{array}$ \\
\hline$[22]$ & Yes & Two & Delay & CSMA & $\begin{array}{l}\text { Dynamically adjustable channel } \\
\text { negotiation Phase Reduce the } \\
\text { bandwidth waste }\end{array}$ & $\begin{array}{l}\text { Cost increase } \\
\text { due to two } \\
\text { transceivers }\end{array}$ \\
\hline
\end{tabular}

\section{CONCLUSIONS}

Cognitive radio technology is a potential technology for future wireless systems like the Internet of Things, WSNs, and M2M systems and provides benefit in coexistence of different wireless technology by improving spectrum utilization. However, when we introduce cognitive capability in WSNs, due to limitation of WSN and to support cognitive capabilities redefinition of protocol stack is required by considering following factors like: the radio environmental, primary user's activities and secondary user's operation limitations such as number of radios, single/multi-band operation, hardware limitation etc. The Cognitive MAC layer and its mechanisms provide a solution to these challenges and improving the secondary user's performance. Many kinds of literature are available for cognitive 
radio network and its MAC protocol but not much survey on CRSN's MAC layer. In this paper, we have presented a brief survey of the different novel design of MAC for CR-WSNs with their pros and cons. According to a survey, we can conclude that the main tasks of cognitive MAC are environment sensing, channel negotiation, and data transmission. We believe our work is helpful for future research

\section{REFERENCES}

1. Han, J.A.; Jeon, W.S.; Jeong, D.G. Energyefficient channel management scheme for cognitive radio sensor networks. IEEE Trans. Veh. Technol. 2011, 60, 1905-1910.

2. Shah, G.A.; Akan, O.B. Ad Hoc Networks Performance analysis of CSMA-based opportunistic medium access protocol in cognitive radio sensor networks. Ad Hoc Netw. 2014, 15, 413.

3. u, Y.; Wu, C.; He, C.; Jiang, L. A Cluster-based Energy Efficient MAC Protocol for Multi-hop Cognitive Radio Sensor Networks. In Proceedings of the IEEE Global Communications Conference, Anaheim, CA, USA, 3-7 December 2012; pp. 537-542.
4. Jamal, A.; Tham, C.; Wong, W.-C. CR-WSN MAC: An Energy Efficient and Spectrum Aware MAC Protocol for Cognitive Radio Sensor Network. In Proceedings of the 9th International Conference on Cognitive Radio Oriented Wireless Networks and Communications (CROWNCOM), Oulu, Finland, 2-4 June 2014; pp. 67-72.

5. .Aijaz, A.; Ping, S.; Akhavan, M.R.; Aghvami, A. CRB-MAC: A receiver-based MAC protocol for cognitive radio equipped smart. IEEE Sens. J. 2014, 14, 4325-4333.

6. Shah, G.A.; Akan, O.B.; Member, S. Cognitive adaptive medium access control in cognitive radio sensor networks. IEEE Trans. Veh. Technol. 2015, 64, 757-767.

7. Glaropoulos, I.; Lagana, M.; Fodor, V.; Petrioli, C. Energy Efficient Cognitive MAC for Sensor Networks under WLAN Co-existence. IEEE Trans. Wirel. Commun. 2015, 14, 1-13.

8. Joshi, G.P.; Nam, S.Y.; Kim, S.W.; Kim, B. Adaptive window size-based medium access control protocol for cognitive radio wireless sensor networks. J. Sens. 2016, 2016, 1-9. 